\title{
Impact of a Porosity-Dependent Retention Function on Simulations of Porous Flow
}

\author{
Peter J. Johnson ${ }^{1,2,3}$ (D) $\cdot$ George A. Zyvoloski ${ }^{1}$ - Philip H. Stauffer ${ }^{1,2}$
}

Received: 15 February 2018 / Accepted: 29 October 2018 / Published online: 9 November 2018

(c) The Author(s) 2018

\begin{abstract}
Numerical models of flow in unsaturated porous media employ a range of functions to account for capillary effects. In general, these retention functions are assigned at the beginning of the simulation and calculate capillary pressure based on saturation. However, many porous systems involve changes in porosity wherein the retention function should change during the simulation. Model runs which neglect these changes may produce unphysical results such as retention of liquid water in air-filled void spaces. We present a conceptually and numerically simple function that recalculates the retention function at each timestep based on the updated porosity. The new retention function updates the maximum capillary pressure, residual saturation, and maximum saturation prior to applying the saturation fit. We compare results from a fixed (saturation-only) function and the new porosity-dependent retention function through a set of two numerical Gedankenexperiments in salt. The new retention function corrects unphysical model behaviors and causes dramatic changes in simulation behavior relative to the fixed (saturation-only) function, especially when applied to systems dominated by capillary effects. These changes result in large differences in simulated porosity, saturation, and volumetric water content. Water content results obtained using the porositydependent retention function are inverted compared to those obtained from saturation-only functions, with high-porosity nodes changing from very wet when using the saturationonly retention function to very dry when using the porosity-dependent retention function. These test cases suggest that dynamic retention functions in changing-porosity systems are important considerations to ensure sensible simulation results.
\end{abstract}

Keywords Capillary $\cdot$ Retention curve $\cdot$ Porosity $\cdot$ Numerical modeling

Philip H. Stauffer

stauffer@lanl.gov

1 EES-16: Computational Earth Science, MS T003, Los Alamos National Laboratory, Los Alamos, NM 87545, USA

2 Department of Geology, The University at Buffalo (SUNY), Amherst, NY 14260, USA

3 Present Address: GNS Science, Wellington, New Zealand 


\section{Lists of symbols}

A Mass per unit volume

$m$ Subscript to denote water phase

$\eta \quad$ Subscript to note air phase

$f \quad$ Flux of subsequent phase

$e \quad$ Subscript to note energy

$q \quad$ Source/sink term

$t \quad$ Time

$S \quad$ Saturation

$\rho \quad$ Density

$v \quad$ Subscript to denote vapor

$l \quad$ Subscript to denote liquid

$\phi \quad$ Porosity

u Volumetric flux term (Darcy flux)

$k \quad$ Permeability

$P \quad$ Pressure

$g \quad$ Gravity vector

$\gamma \quad$ Specific internal energy for subscripted phase

$C_{\mathrm{pr}} \quad$ Specific heat capacity of rock

$T$ Temperature

$h \quad$ Specific enthalpy

$\kappa \quad$ Thermal conductivity

$R_{1} \quad$ Liquid relative permeability

$S_{1} \quad$ Liquid saturation

$S_{\operatorname{lmax}}$ Maximum saturation above which capillary pressure goes to 0

$S_{\mathrm{r}} \quad$ Residual saturation

$\kappa_{\mathrm{t}} \quad$ Temperature-dependent thermal conductivity

ic Subscript denoting chemistry timestep

$\Delta c \quad$ Change in moles of solid salt per weight of the solid

$m_{\mathrm{s}} \quad$ Molar mass of solid

$D_{\text {va }} \quad$ Vapor diffusion

$\phi_{\mathrm{i}} \quad$ Initial porosity

$\tau \quad$ Tortuosity

$S_{\mathrm{v}} \quad$ Air saturation with respect to water vapor

$f_{\text {mwv }}$ Mass flux of water vapor

$P_{\mathrm{c}} \quad$ Calculated capillary pressure

$W_{\text {Mwv }}$ Molecular weight of water vapor

$P_{\text {cmax }} \quad$ Maximum capillary pressure

$P_{\text {cmaxi }}$ User-specified initial maximum capillary pressure

$S_{\text {ri }} \quad$ User-specified initial residual saturation

$Q \quad$ Empirical exponent for Buckles (1965) residual saturation function

$V_{1} \quad$ Liquid specific volume 


\section{Introduction}

Porosity changes are common in unsaturated porous media. Examples include industrial applications such as personal hygiene tissues (e.g., Sun et al. 2015), absorbent polymers (e.g., Brandt et al. 1987), cooling systems (e.g., Jo et al. 2018), and many others. Geological systems with changing porosity are abundant and include mineral dissolution or precipitation in karst, geothermal, or hydrothermal systems (e.g., Evans and Lizarralde 2003; Waltham et al. 2005; Ball et al. 2015), thermal or mechanical stress, (e.g., Tsang 1999), bioturbation (e.g., Pérès et al. 1998; Gingras et al. 2012), diagenetic processes (e.g., Gluyas and Coleman 1992), simple compaction of deposits (Boudreau and Bennett 1999), and radiogenic waste in salt (e.g., Jordan et al. 2015a, b, c; Bourret et al. 2017). Research conducted in these areas often includes numerical modeling in the unsaturated zone.

Many numerical models of unsaturated flow employ some form of user-defined retention function which is specified at the beginning of the simulation. Capillary pressure is then determined from the local saturation and is used to calculate fluid flow. Most commonly used models follow this broad approach, such as Hydrus (Šimůnek et al. 2012), TOUGH2 (Doughty 2013; http://esd1.lbl.gov/research/projects/tough/; c.f. Calore and Battistelli 2003), OpenGeoSys (Kolditz et al. 2012; Wang et al. 2015), PFLOTRAN (Lichtner et al. 2015), and others. Several different formulations are commonly employed in these models including linear functions, the Brooks and Corey (1964) functions, and the van Genuchten (1980) functions. Most of these functions are designed to work with a single soil/rock type throughout a simulation, referred to herein as a fixed or saturation-only retention function. However, using a single, saturation-only form can become problematic if porosity changes from the initial condition. As porosity changes, the original retention function may no longer be representative of the new local porous medium. Consider an end member thought experiment example that clearly demonstrates incorrect model behavior: Imagine that a region of the model domain completely dissolves, attaining porosity $=1$; if the retention function is not changed from its original specification, this region will maintain a nonzero capillary pressure to try to satisfy the saturation-pressure balance. In this situation, water will tend to be retained in what is effectively open air. Gravity should cause this retained water to drain from the porosity $=1$ region, but the original retention function, appropriate for the initial porosity, prevents drainage.

In this paper, we describe a retention function developed to dynamically alter the capillary pressure as a function of saturation based on the updated porosity at each timestep. The function is designed to be conceptually and numerically simple to facilitate application to complex problems where model convergence may be difficult independent of the retention function. Equations are implemented in the Los Alamos National Laboratory (LANL) developed porous flow simulator FEHM (Finite Element Heat and Mass Transfer Code; Zyvoloski et al. 2012) and applied to two simple thought experiments in order to demonstrate the effects of modifying the retention function as a result of changing porosity.

\section{Background}

Porous flow simulators typically allow the user to choose from a variety of standard retention functions (e.g., linear, van Genuchten, Brooks-Corey) that vary capillary pressure $\left(P_{\mathrm{c}}\right)$ as a function of liquid saturation $\left(S_{1}\right)$. The specifics of function implementation in different models vary, but the overall structure is generally similar. At some low saturation, variously 
referred to as residual saturation, irreducible saturation, or critical saturation, $P_{\mathrm{c}}$ rises rapidly to some maximum value $\left(P_{\mathrm{cmax}}\right)$. We here refer to this low saturation end member as the residual saturation $\left(S_{\mathrm{r}}\right)$. The other endpoint of interest is a maximum liquid saturation, $S_{\operatorname{lmax}}$, above which capillary pressure goes to zero. A linear or fit is then applied between these two points to generate a retention function or characteristic curve.

Several studies have considered capillary effects with changing porosity. Leverett (1941) developed a dimensionless parameter which, when plotted against saturation, produced a single curve for a material type. This dimensionless parameter included capillary pressure and effectively a capillary radius term as the square root of $k / \phi$, where $k$ is permeability in $\mathrm{m}^{2}$ and $\phi$ is porosity. In this function, capillary pressure and permeability thus alter in response to porosity changes. A form of this theory has been implemented in the modeling software TOUGH2 (Fakcharoenphol et al. 2013),

$$
P_{\mathrm{c}}=P_{\mathrm{co}} \frac{(\sqrt{k / \phi})_{0}}{\sqrt{k / \phi}},
$$

but without dynamic adjustment of the end members and with a single function of saturation applied to the resulting capillary pressure calculation. Numerous experiments have shown that capillary pressure of the wetting phase is stronger when porosity is reduced (e.g., Gallipoli et al. 2003; Nuth and Laloui 2008; Oh and Lu 2014). Salager et al. (2010) conducted experiments on soil and developed a three-dimensional surface of saturation, porosity, and capillary pressure that showed increasing strength of capillary effects for equal saturation as porosity decreased. Conversely, capillary effects decrease as pore diameters increase. Open air, with a porosity $\phi=1$, has no capillary pressure because there is no solid grain on which fluid-solid surface interactions can apply. Sweijen et al. (2016) used combined discrete element and pore unit modeling on absorbent gel particles to generate van Genuchten curves for porosities ranging from 0.1 to 0.5 . For porosities above this range, the precise nature of the decrease in pressures as porosity increases is unclear, but general trends can be identified. Theory using capillary tube or spherical grain models (c.f. Lucas 1918; Washburn 1921; Finn 1999; Masoodi and Pillai 2012; Sweijen et al. 2016) allows for a general intuition of the capillary changes with porosity. Functions for specific materials depend on the pore geometry and the nature of the fluid-solid contact (e.g., contact angle) which controls the spreading of the wetting phase across the surface of the solid medium. For bundled cylindrical tubes with a fixed radius, Masoodi and Pillai (2012) derived a general relationship based on the Young-Laplace equation as

$$
P_{\mathrm{c}}=2 \frac{\epsilon_{1} \cos \theta}{r_{\mathrm{c}}}
$$

where $\epsilon$ is the surface tension of the fluid $(\mathrm{N} / \mathrm{m}), \theta$ is the contact angle between the fluid and solid, and $r_{\mathrm{c}}$ is the tube radius $(\mathrm{m})$. If porosity increases are caused by expanding pores rather than the formation of new pores, $r_{\mathrm{c}}$ will also increase, driving capillary pressure toward zero. Likewise, for spherical particles, Masoodi and Pillai (2012) derived the capillary pressure as

$$
P_{\mathrm{c}}=3 \frac{1-\phi}{\phi} \frac{\epsilon_{1} \cos \theta}{r_{\mathrm{sp}}}
$$

where $r_{\mathrm{sp}}$ is the equivalent spherical radius. In this system, too, capillary pressure drops as porosity increases and also as the radius of spherical particles increases due to the increased diameter of intergranular void spaces. This provides guidance on how capillary pressure should change within the model even for porosities greater than 0.5 , the upper limit examined by Sweijen et al. (2016). 
The new retention function we describe in this paper makes an assumption of a consistent composition and structure of the porous medium surrounding a pore, so that parameters such as contact angle and pore shape are unchanging. Capillary differences therefore arise only from increasing or decreasing pore diameter. In the salt scenarios considered for this work, pore diameter is assumed to change as pores expand or contract when salt dissolves or precipitates at the perimeter of the pore. Note that this approach would require modification in systems where porosity changes include changes in the mineral phase at the pore throat margin (e.g., formation of caliche horizons in silicate soils) or where porosity is increased by formation of new pores instead of widening existing pores.

We base our porosity-dependent retention function on a simple linear retention function previously implemented within FEHM, but a similar approach could be applied to the Brooks-Corey and van Genuchten functions as well. The general form of the linear retention function is

$$
\begin{aligned}
& P_{\mathrm{c}}=P_{\mathrm{cmax}}, \quad S_{1} \leq S_{\mathrm{r}} \\
& P_{\mathrm{c}}=P_{\mathrm{cmax}} \frac{S_{\mathrm{lmax}}-S_{\mathrm{l}}}{S_{\operatorname{lmax}}-S_{\mathrm{r}}}, \quad S_{\mathrm{r}}<S_{1}<S_{\mathrm{lmax}} \\
& P_{\mathrm{c}}=0, \quad S_{1} \geq S_{\mathrm{lmax}}
\end{aligned}
$$

where $P_{\mathrm{c}}$ is the calculated capillary pressure, $P_{\mathrm{cmax}}$ is the specified maximum capillary pressure value, $S_{\mathrm{r}}$ is the residual saturation, $S_{1}$ is current liquid saturation, and $S_{\text {lmax }}$ is the saturation at or above which capillary pressure is zero. This function is chosen for its conceptual simplicity and also for a reduction in numerical complexity, allowing application of the new porosity-dependent function to complex multiphase heat/stress/mass flow/chemical domains where model convergence may already be challenging.

\section{Porous Flow Simulator}

The present work is motivated by ongoing modeling efforts in support of radioactive waste disposal in salt. In the interest of completeness, this section describes the numerical porous flow simulator, including the salt equations which have not been previously presented in their current form in the published literature. We apply the new retention function within FEHM through code changes to appropriate subroutines (Zyvoloski et al. 2012; FEHM 2017). FEHM has been used to simulate a wide variety of multiphase coupled heat/stress/mass flow and transport problems (e.g., Stauffer et al. 1997, 2005; Yamaguchi et al. 1991; Spinelli and Fisher 2004; Fisher and von Herzen 2005; Tenma et al. 2008; Winslow et al. 2016; Birdsell et al. 2000; Arnold et al. 2003; Kelkar et al. 2013). FEHM, formulated primarily as a finite volume simulator, applies equations for conservation of mass and energy between connected volumes as:

conservation of water mass,

$$
\frac{\partial A_{\mathrm{m}}}{\partial t}+\nabla \cdot \boldsymbol{f}_{\mathrm{m}}+q_{\mathrm{m}}=0
$$

conservation of air mass,

$$
\frac{\partial A_{\eta}}{\partial t}+\nabla \cdot \boldsymbol{f}_{\eta}+q_{\eta}=0,
$$

and conservation of energy,

$$
\frac{\partial A_{\mathrm{e}}}{\partial t}+\nabla \cdot \boldsymbol{f}_{\mathrm{e}}+q_{\mathrm{e}}=0
$$


where $A(m, \eta)$ are the mass per unit volume of water and air, respectively; $A_{\mathrm{e}}$ is similarly the energy per unit volume; $\boldsymbol{f}(m, \eta)$ are water and air mass fluxes with units of mass per area time while $f(e)$ is energy flux, with units of energy per area time. Finally, $q$ is the source/sink term and $t$ is time. Water mass per unit volume $A_{\mathrm{m}}$ is given by

$$
A_{\mathrm{m}}=\phi\left(S_{\mathrm{v}} \rho_{\mathrm{v}}\left(1-\eta_{\mathrm{v}}\right)+S_{1} \rho_{\mathrm{l}}\left(1-\eta_{1}\right)\right)
$$

where $S$ is the saturation and $\rho$ the density of the vapor phase and liquid phase (subscripts $v$ and $l$, respectively); $\phi$ is porosity; and $\eta$ is the mass fraction of air contained in the vapor phase. Air mass per unit volume is similarly,

$$
A_{\eta}=\phi\left(S_{\mathrm{v}} \rho_{\mathrm{v}} \eta_{\mathrm{v}}+S_{1} \rho_{1} \eta_{1}\right)
$$

Mass fluxes for water and air are:

$$
f_{\mathrm{m}}=\left(1-\eta_{\mathrm{v}}\right) \rho_{\mathrm{v}} \boldsymbol{u}_{\mathrm{v}}+\left(1-\eta_{1}\right) \rho_{\mathrm{l}} \boldsymbol{u}_{1}
$$

and

$$
f_{\eta}=\phi\left(\eta_{\mathrm{v}} \rho_{\mathrm{v}} \boldsymbol{u}_{\mathrm{v}}+S_{\mathrm{l}} \rho_{\mathrm{l}} \boldsymbol{u}_{1}\right)
$$

where $\boldsymbol{u}$ is the volumetric flux, variously known as Darcy flux, specific discharge, fictitious velocity, etc. (Stauffer 2006). Darcy's Law applies to the movement of the vapor and liquid,

$$
\boldsymbol{u}_{\mathrm{v}}=-\frac{k_{\mathrm{rv}}}{\mu_{\mathrm{v}}}\left(\nabla P_{\mathrm{v}}-\rho_{\mathrm{v}} g\right)
$$

and

$$
\boldsymbol{u}_{1}=-\frac{k_{\mathrm{rl}}}{\mu_{1}}\left(\nabla P_{1}-\rho_{1} g\right)
$$

where $k_{\mathrm{r}}$ is the relative permeability of the respective phases, $P$ is pressure, and $g$ is the gravitational vector.

Energy per unit volume $A_{\mathrm{e}}$ is

$$
A_{\mathrm{e}}=(1-\phi) \rho_{\mathrm{r}} \gamma_{\mathrm{r}}+\phi\left(S_{\mathrm{v}} \rho_{\mathrm{v}} \gamma_{\mathrm{v}}+S_{\mathrm{l}} \rho_{\mathrm{l}} \gamma_{\mathrm{l}}\right)
$$

with $\gamma_{\mathrm{r}}=C_{\mathrm{pr}} T$, and the energy flux $f_{\mathrm{e}}$ given by

$$
f_{\mathrm{e}}=\rho_{\mathrm{v}} h_{\mathrm{v}} \boldsymbol{u}_{\mathrm{v}}+\rho_{\mathrm{l}} h_{1} \boldsymbol{u}_{1}-\kappa \nabla T
$$

The subscript $r$ refers to the solid matrix; $\gamma$ is the specific internal energy for each respective phase; $C_{\mathrm{pr}}$ is specific heat; $h_{\mathrm{v}}$ and $h_{1}$ are specific enthalpies; $\kappa$ is effective thermal conductivity; and $T$ is temperature. Gravitational potential energy is embedded in the liquid phase specific enthalpy definition (Stauffer et al. 2014b) in FEHM as,

$$
h_{1}=C_{\mathrm{p}} T+P_{1} V_{1}+g z
$$

where $C_{\mathrm{p}}$ is the heat capacity, $T$ temperature, $P$ pressure, $V_{1}$ is specific volume, and $z$ is height above a reference in the direction away from the center of mass of the gravity field. With the inclusion of potential energy, the enthalpy term is sometimes referred to as methalpy (Stauffer et al. 2014b).

Fluid density and viscosity are expressed as polynomial functions of pressure and temperature and are fit to National Bureau of Standards data (Haar et al. 1984). For mixed air/water phases within an element, the relative permeability $R_{1}$ function is a linear fit given by 


$$
R_{\mathrm{l}}=\frac{S_{\mathrm{l}}-S_{\mathrm{r}}}{S_{\mathrm{lmax}}-S_{\mathrm{r}}}
$$

where $S_{1}$ is liquid saturation and $S_{\mathrm{r}}$ is residual saturation. Other formulations of the relative permeability function are available in FEHM, but only the linear approach is implemented for the porosity-dependent retention function at the present time.

The final set of constitutive relationships concern the governing equations for dissolution, precipitation, and material properties for the porous medium. For the work described herein, the porous medium is considered as salt (pure halite) using model functions applied to previous work at the Waste Isolation Pilot Plant (WIPP; e.g., Stauffer et al. 2013, 2014a; Harp et al. 2014; Bourret et al. 2016, 2017; Johnson et al. 2017). The FEHM salt functions account for the solubility with temperature of salt in brine, such that change in temperature and moisture content produce precipitation or dissolution of the porous medium that are linked to porosity and permeability changes. In addition, the salt subroutines account for temporal changes in the salt medium rock properties. These salt functions have previously been developed and tested (Stauffer et al. 2013; Harp et al. 2014; Jordan et al. 2015a, b, c; Bourret et al. 2017) and have induced major changes in porosity in the simulated domains when three phase (brine, vapor, salt) heat pipes develop.

Munson et al. (1990) identified a temperature dependence of thermal conductivity of intact salt, $\kappa(T)$, as

$$
\kappa_{T-\mathrm{WIP} P}(T)=\kappa_{T-300}\left(\frac{300}{T}\right)^{1.14}
$$

where $T$ is temperature in kelvin and $\kappa_{T-300}$ is the thermal conductivity of intact salt at $300 \mathrm{~K}$ $\left(5.4 \mathrm{~W} \mathrm{~m}^{-1} \mathrm{~K}^{-1}\right)$. Thermal conductivity is also dependent on porosity $n$ and was described by Gable et al. (2009), based on work inverse modeling of heat experiments conducted by Bechtold et al. (2004), at the Asse salt mine in Germany as

$$
\kappa_{\mathrm{T}-\operatorname{ASSE}}(\phi)=-270 \phi^{4}+370 \phi^{3}-136 \phi^{2}+1.5 \phi+5
$$

A modification of this function is required for higher porosities because the fourth-order polynomial deviates from physical sensibility when $\phi>0.4$, first increasing from $0.4<\phi<0.6$ and then decreasing rapidly to become negative at $n \phi>0.75$. Consequently, a truncation is applied to this function in the present implementation of FEHM in which thermal conductivity for $\phi>0.4$ is held constant at the polynomial local minimum value. Temperature and porosity effects on thermal conductivity are combined by scaling $\kappa_{\mathrm{T}-\mathrm{ASSE}}$ to match $\kappa_{\mathrm{T}-300}$ at $\phi=0$,

$$
\kappa_{\mathrm{T}-300}(\phi)=\left(\frac{\kappa_{\mathrm{T}-300}}{\kappa_{\mathrm{T}-\mathrm{ASSE}}(\phi=0)}\right) \times \kappa_{\mathrm{T}-\operatorname{ASSE}}(\phi)
$$

In this case, $\kappa_{\mathrm{T}-300} / \kappa_{\mathrm{T}-\mathrm{ASSE}}$ is $5.4 / 5.0$ or 1.08 .

Solubility of salt in water is a function of temperature derived from Sparrow (2003). In the modeled range of temperatures, solubility ranges from about 6.1 to $6.8 \mathrm{~mol} / \mathrm{kg}$. The code calculates precipitation and dissolution of a tracer to which salt properties are assigned. We assume that the solid matrix is salt, and that any calculated precipitation or dissolution results in a porosity change. The tracer function includes separate terms for the solid matrix and the tracer within the liquid phase; this formulation is applied to allow flexibility in applying a single tracer macro-form to multiple problems. Thus, after calculating a volume change due to precipitation or dissolution, the total $\mathrm{mol} / \mathrm{kg}$ of the solid phase is forced to remain constant at the value of solid salt. For the salt function, the tracer is chemically the same as the matrix, so the change in tracer concentration between liquid and solid phases describes the 
dissolution/precipitation of the salt. Precipitation of salt fills pore space, while dissolution increases pore space; porosity is therefore related directly to porosity in each chemistry iteration ic as

$$
\Delta \phi_{\mathrm{ic}}=-\Delta c \rho_{\mathrm{s}} \mu_{\mathrm{s}} \frac{1}{\rho_{\mathrm{NaCl}}}\left(1-\phi_{\mathrm{ic}}\right)
$$

where $\Delta c$ is the change of moles of solid salt per weight of the solid $(\mathrm{kg}), \rho_{\mathrm{s}}$ is density of the solid $\left(\mathrm{kg} / \mathrm{m}^{3}\right), \mu_{\mathrm{S}}$ is the molar mass of the solid $(\mathrm{kg} / \mathrm{mol})$, and $\rho_{\mathrm{NaCl}}$ is the density of salt, with $\rho_{\mathrm{s}}=\rho_{\mathrm{NaCl}}$. Permeability is related to porosity linearly as described by Cinar et al. (2006). For numerical stability in the current function, porosity is constrained to lie between a maximum of 0.9999 and a minimum of $10^{-5}$. We note that Eq. 21 here places a negative before Eq. 6 of Stauffer et al. (2013), correcting this equation to produce a decrease in porosity as the concentration of the solid phase increases.

Water vapor diffusion accounts for thermal and pressure effects described by

$$
D_{\mathrm{va}}=\tau D_{\mathrm{va}}^{\mathrm{o}}\left(P_{\mathrm{o}} / P\right)\left(\frac{T+T_{\mathrm{o}}}{T_{\mathrm{o}}}\right) 1.81
$$

where $\tau$ is tortuosity, $D_{\text {va }}^{\mathrm{o}}=2.23 \times 10^{-5}, T_{\mathrm{o}}=273.15 \mathrm{~K}, T$ is temperature $\left({ }^{\circ} \mathrm{C}\right), P$ is pressure (MPa), and $P_{\mathrm{o}}=0.1 \mathrm{MPa}$ (Pruess 1991). The effective free air water vapor diffusion coefficient is then modified based on the Millington and Quirk (1961) relationship. This relationship is commonly applied as a simple gradient term based on Fick's first law,

$$
\boldsymbol{J}=D_{\text {eff }} \nabla C
$$

where $\boldsymbol{J}$ is the solute flux $\left(\mathrm{mol} / \mathrm{m}^{2} \mathrm{~s}\right)$ and $\mathrm{C}$ is concentration $(\mathrm{mol} / \mathrm{L})$. The effective diffusivity $D_{\text {eff }}$ is dependent on porosity and non-liquid fraction of the pore space, or air content $\theta_{\mathrm{a}}=$ $S_{\mathrm{a}} n$, where $S_{\mathrm{a}}$ is air saturation, $1-S_{1}$ (Jury and Gardner 1991):

$$
D_{\text {eff }}=\frac{D_{\text {free }} \theta_{\mathrm{a}}^{10 / 3}}{\phi^{2}}
$$

Combining the effective and free diffusivity terms into a tortuosity term, $\tau$, yields

$$
\tau=\frac{\left(S_{\mathrm{a}} \phi\right)^{10 / 3}}{\phi^{2}}
$$

However, many porous media simulators, including FEHM, use a modification of this diffusivity for porous media (Ho and Webb 1998),

$$
D^{*}=\frac{D_{\text {eff }}}{S_{\mathrm{a}} \phi}
$$

In practice, this changes the exponent in the tortuosity term from 10/3, as expressed in Eq. 25, to

$$
\tau=\left(S_{\mathrm{a}} \phi\right)^{7 / 3} / \phi^{2}
$$

The measured diffusion value using the simple $D_{\text {eff }}$ formulation must be increased by $1 / \theta_{\text {a }}$ for use in most transient modeling, a point that is often missed and can cause an underestimate of diffusivity in modeling work when porous media properties are not included (Stauffer et al. 2009). Mass transport for diffusion of water vapor through air is driven by a concentration gradient in the bulk vapor phase (air + water vapor) as

$$
f_{\mathrm{mwv}}=-D_{\mathrm{va}} \phi S_{\mathrm{v}} W_{\mathrm{Mwv}} \nabla C_{\mathrm{wv}}
$$


where $f_{\mathrm{mwv}}$ is the diffusive mass flux of water vapor, $W_{\mathrm{Mwv}}$ is the molecular weight of water vapor $(\mathrm{kg} / \mathrm{mol})$, and $C_{\mathrm{wv}}$ is the moles of water vapor per cubic meter. Similarly, the noncondensible fraction of the bulk vapor phase (air) is allowed to diffuse along its concentration gradient with a diffusive mass flux as:

$$
f_{\mathrm{ma}}=-D_{\mathrm{va}} \phi S_{\mathrm{v}} W_{\mathrm{Mwa}} \nabla C_{\mathrm{a}}
$$

where $f_{\text {mwa }}$ is the diffusive mass flux of air, $W_{\text {Ma }}$ is the molecular weight of air $(\mathrm{kg} / \mathrm{mol})$, and $C_{\mathrm{wa}}$ is the moles of air per cubic meter. We note that a common practice in porous flow simulators, assuming binary diffusion where $f_{\mathrm{ma}}=\boldsymbol{f}_{\mathrm{mwv}}$, can lead to non-physical model behavior and we recommend that both terms be independently calculated. This is because, in the presence of a temperature gradient, the condensable flux may not remain in the vapor phase but can condense into the liquid phase.

\section{Retention as a Function of Porosity and Saturation: Linear Formulation}

The overall objective of our new retention function is to produce a varying capillary pressure curve as a function of saturation for modeling of porous media with changing porosity. We therefore aim to produce stronger capillary pressure effects as porosity decreases and weaker effects as porosity increases. This is done by adjusting the residual saturation $\left(S_{\mathrm{r}}\right)$ and maximum capillary pressure at low saturations $\left(P_{\mathrm{cmax}}\right)$ values and then recalculating the retention function at each timestep (Fig. 1). The user inputs the initial parameters for conditions at the start of the simulation. Subsequent timesteps read the updated node porosity from model global variables and calculate an updated retention function at each node.

\subsection{Residual Saturation}

In open air $(\phi=1)$, residual saturation is 0 and there is no capillary pressure because there is no solid grain on which wetting can occur. As porosity decreases, $S_{\mathrm{r}}$ increases. Sweijen et al. (2016) showed a weak dependency of $S_{\mathrm{r}}$ on porosity for $0.1<\phi<0.5$, but could not examine higher or lower porosity values due to their approach using packed spherical particles. Their fitted relationship was given as:

$$
S_{\mathrm{r}}=-1.7 \phi^{2}+\phi
$$

This relationship produces negative values at porosities above about 0.588 , a consequence of the spherical model constraints used in that work. The Sweijen et al. (2016) relationship also causes a reduction in residual saturation for porosities less than about 0.294 , implying that more complete drainage of porous media becomes easier as porosity reduces which is generally not the case if pore characteristics other than diameter remain consistent. Conversely, Buckles (1965) and subsequent work by Holmes et al. (2009) proposed a different relationship,

$$
\phi^{\mathrm{Q}} S_{\mathrm{r}}=\text { constant }
$$

where $Q$ is specific to the material but generally falls close to 1 . Solving for $S_{\mathrm{r}}$ yields a curve that greatly increases the residual saturation as porosity approaches 0 , until $S_{\mathrm{r}}$ eventually surpasses 1 . Some form of truncation to this function would be necessary to prevent unphysical residual saturation. Furthermore, this function induces steep pressure gradients as porosity 


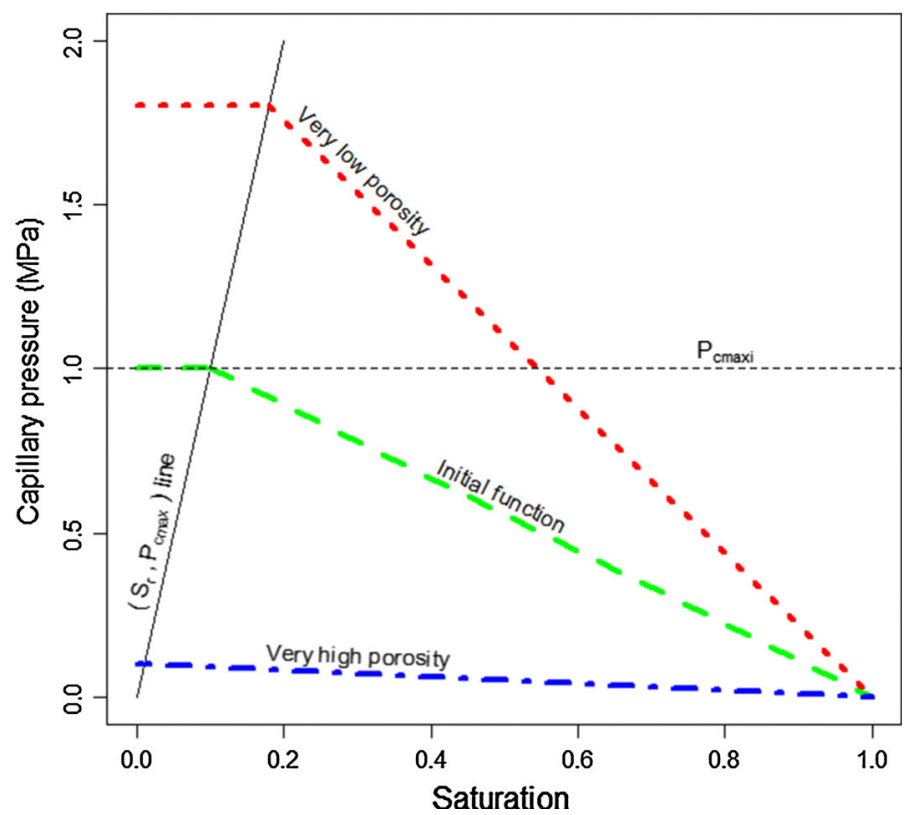

Fig. 1 Example of updating the retention function. The user specifies values for initial condition (residual saturation $S_{\text {ri }}$ of 0.1 , maximum capillary pressure $P_{\text {cmaxi }}$ of $0.3 \mathrm{MPa}$ ). Residual saturation and maximum capillary pressure are then recalculated based on a linear extrapolation from $(0,0)$ through the initially specified point. High-porosity nodes then have generally very low capillary pressures, while low-porosity nodes have very high capillary pressures

approaches 0 , and at very low porosity the function becomes nearly vertical so that small changes in saturation cause large differences in capillary pressure. This can present challenges to model solver routines because minor changes made to the incoming solution in each new iteration can cause instability. Depending on the specifics of the solver routines and model design, this can result in greatly increased numbers of iterations per timestep, reduction in size of timesteps, and overall a substantial increase in runtime. In some extreme cases the instability can cause the simulation to fail to reach convergence.

In order to force a changing residual saturation while avoiding the low-porosity solver convergence issues of the Buckles (1965) formula, we apply a linear function of residual saturation with respect to porosity,

$$
S_{\mathrm{r}}(\phi)=S_{\mathrm{ri}} \frac{1-\phi}{1-\phi_{\mathrm{i}}} .
$$

This yields the appropriate residual saturation for the user-specified porosity and $S_{\mathrm{r}}$ of 0 at $\phi=1$, and produces an increase in residual saturation with decreasing porosity. The linear interpolation tends to approximately match the Buckles (1965) and Sweijen et al. (2016) functions for mid-range porosities (Fig. 2). The linear function avoids taking unphysical negative values at higher porosity, as in the Sweijen et al. (2016) function. The linear function tends to underestimate low-porosity residual saturation values compared to the Buckles (1965) formulation but nevertheless produces stronger capillary effects for equal saturation in the decreasing porosity case. 


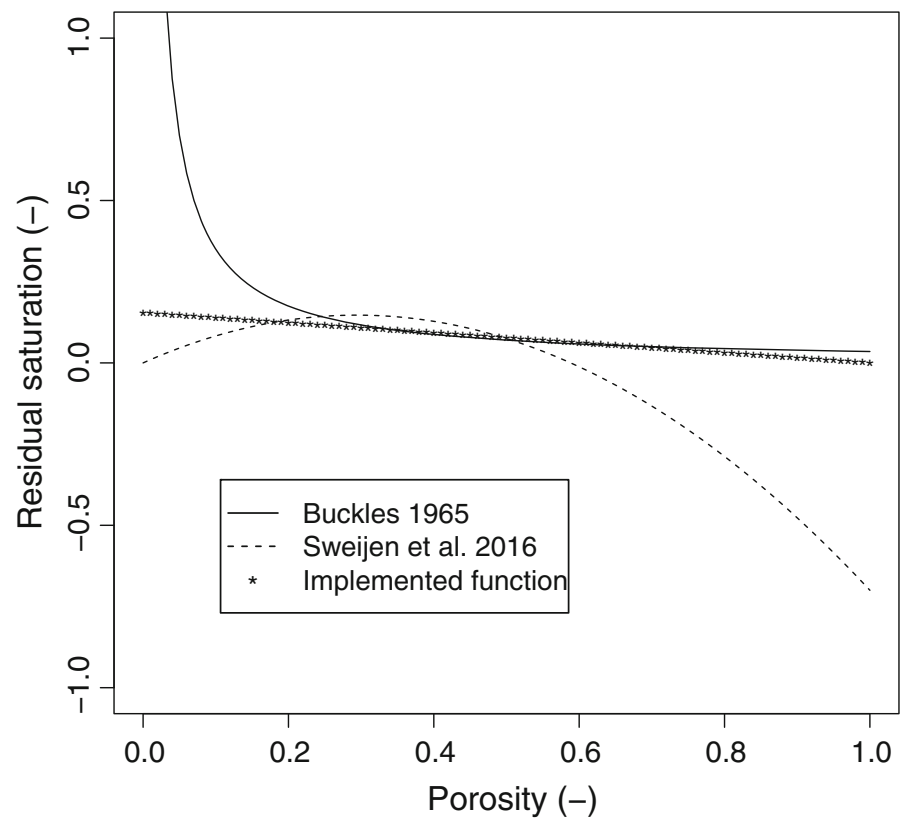

Fig. 2 Residual saturation $\left(S_{\mathrm{r}}\right)$ as a function of porosity (n). Equation fit (Eq. 4) based on Sweijen et al. (2016) is shown with solid line; dashed line is function (Eq. 5) from Buckles (1965); dotted line is chosen linear function. The linear function closely matches Buckles for porosity $>0.3$ and forces increased $S_{\mathrm{r}}$ at low porosities while avoiding the steep gradient and resultant convergence issues

\subsection{Retention Function}

Maximum capillary pressure will increase as pore throat diameter decreases. Similarly to residual saturation, we apply a linear fit to this value which extrapolates from $P_{\mathrm{cmax}}$ of 0 at $\phi$ $=1$ through the user supplied value $P_{\mathrm{cmaxi}}$ at $\phi_{i}$ to a variable calculated $\phi=0$. In this case, the upper constraints of both $P_{\mathrm{cmax}}$ and the maximum residual saturation are not supplied by the user but instead determined within the model. $P_{\text {cmax }}$ is calculated as

$$
P_{\mathrm{cmax}}=P_{\mathrm{cmaxi}}\left(\frac{1-\phi}{1-\phi_{\mathrm{i}}}\right)
$$

In combination, the adjusted $S_{\mathrm{r}}$ and $P_{\text {cmax }}$ functions lead to higher capillary pressures at higher saturations when porosity decreases, and the inverse as porosity increases.

The other end point of the retention function is the saturation above which capillary forces are zero, $S_{\operatorname{lmax}} . S_{\operatorname{lmax}}$ is highly dependent on the specifics of the porous medium, and a generalized formulation is difficult. For simplicity, this value is currently held at 1 , but the variable is included in subsequent equations to allow for flexibility in implementation. With these endpoints established, the function is assigned as in Eq. 3 above, but with dynamic variation of the endpoints: 
Table 1 Salt properties input as initial conditions for example problems

\begin{tabular}{lll}
\hline Property & Value & Units \\
\hline Solid density & 2165.0 & $\mathrm{~kg} / \mathrm{m}^{3}$ \\
Specific heat capacity & 931.0 & $\mathrm{~J} / \mathrm{kg} \mathrm{K}$ \\
Permeability & $1 \times 10^{-12}$ & $\mathrm{~m}^{2}$ \\
Thermal conductivity & 1.1 & $\mathrm{~W} / \mathrm{m} \mathrm{K}$ \\
Porosity & 0.3 & - \\
\hline
\end{tabular}

$$
\begin{aligned}
P_{\mathrm{c}} & =P_{\mathrm{cmaxi}}\left(\frac{1-\phi}{1-\phi_{\mathrm{i}}}\right), \quad S_{\mathrm{l}} \leq S_{\mathrm{ri}} \frac{1-\phi}{1-\phi_{\mathrm{i}}} \\
P_{\mathrm{c}} & =P_{\mathrm{cmax}} \frac{S_{\mathrm{lmax}}-S_{l}}{S_{\mathrm{lmax}}-S_{\mathrm{ri}} \frac{1-\phi}{1-\phi_{i}}}, \quad S_{\mathrm{ri}} \frac{1-\phi}{1-\phi_{\mathrm{i}}}<S_{1}<S_{\operatorname{lmax}} \\
P_{\mathrm{c}} & =0.0, \quad S_{1} \geq S_{\mathrm{lmax}}
\end{aligned}
$$

By keeping $P_{\text {cmax }}, S_{\mathrm{r}}$, and $S_{\operatorname{lmax}}$ as separate calculations within the code, the retention function can be easily altered at a later time to allow changes in how these variables are calculated.

\section{Thought Experiment Examples}

To demonstrate the effects of changing the retention function with porosity, we consider two numerical thought experiments in which vigorous liquid, vapor, and solute transport lead to changes in porosity. We further design these domains to feature an extensive unsaturated zone and strong capillary effects. Both domains are designed to induce large porosity changes due to high temperature gradients, the relatively high differences in solubility of salt with temperature, and abundant, continuous phase changes of brine. In the first example, we use a simple $1 \mathrm{~m}$ wide, $0.3 \mathrm{~m}$ tall 2-D radial cylinder which is heated at one end and cooled at the other similarly to the salt cylinder experiments of Olivella et al. (2011), but scaled up an order of magnitude. Gravity is disabled for this first example. For the second example, a $10 \mathrm{~m} \times 10 \mathrm{~m}$ square domain is used with cold top and right-hand boundaries and a heat source applied near the lower left corner. This temperature field induces convective flow of brine and vapor, gravity drainage and capillary flow, and leads to a more complex interaction between phases. Both example problems use material properties generally informed by crushed salt (Table 1). Together, these numerical thought experiments show that large differences in results occur when porosity effects are considered compared to when only an initial, single retention function is specified.

\subsection{Heat Pipe}

The first domain is a $1 \mathrm{~m}$ long by $0.3 \mathrm{~m}$ tall 2-D cylinder with uniform $0.1 \mathrm{~m}$ grid spacing (Fig. 3). Temperature is initially specified at a uniform $20^{\circ} \mathrm{C}$. Note that salt capillary pressure can be quite high; Cinar et al. (2006) measured capillary pressures of $>0.5 \mathrm{MPa}$ in wellsorted, granular salt, so a $1 \mathrm{MPa}$ maximum value is assigned for a potentially poorly sorted salt domain. A constant temperature of $110^{\circ} \mathrm{C}$ is applied to the $x=0 \mathrm{~m}$ (left) boundary and $5^{\circ} \mathrm{C}$ at the $x=1 \mathrm{~m}$ (right) boundary. The top and bottom of the cylinder are perfect insulators 


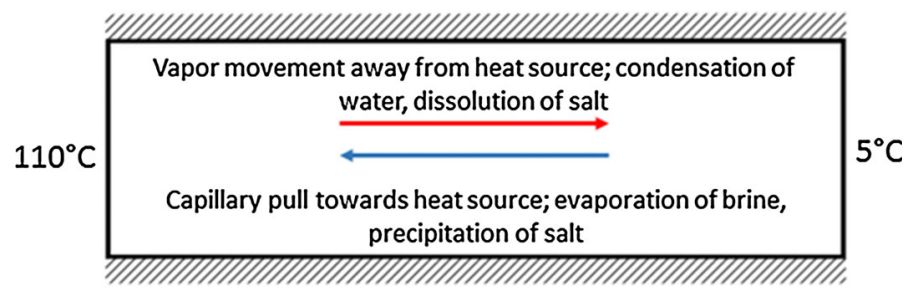

Fig. 3 Salt cylinder domain and conceptual model. Top and bottom are no-flow, insulated boundaries. Fluid may not pass through the left or right boundaries

and all edges are no-flow boundaries with respect to fluid flow. No sources or sinks for water are applied, with only an initial saturation of 0.5 for all nodes providing moisture. The model is run for 365 days of model time. Specifying the domain in this manner induces brine and vapor migration that causes porosity changes through dissolution and precipitation. This process has been the subject of recent attention (c.f. Doughty and Pruess 1990; Birkholzer 2004; Cinar et al. 2006; Olivella et al. 2011; Caporuscio et al. 2013; Kuhlman and Malama 2013; Jordan et al. 2015a, b, c; Stauffer et al. 2014a, b; Rutqvist et al. 2016; Bourret et al. 2017) due to the consideration of salt as a host rock for radiogenic waste. A comprehensive discussion of brine migration in salt is beyond the scope of this paper, but a brief description of the physical mechanisms as relevant to the current work follows.

Evaporation occurs at and near the heat source, oversaturating the remaining brine with respect to salt and inducing mineral precipitation. This precipitated salt reduces porosity and permeability near the heat source, leading to higher capillary pressure. Water vapor transports away from the heat source toward the cold end of the cylinder. As it does, it cools and condenses which increases the available volume of unsaturated water in cooler nodes, inducing dissolution and an increase in porosity and permeability leading to lower capillary pressure. Condensation is driven by changing water vapor pressure, the curve of which is steepest at high temperatures and is depressed by the presence of salt to about $75 \%$ of its desalinated value (Fig. 4; Bourret et al. 2017). The boiling point of saline brine is raised to about $108.5^{\circ} \mathrm{C}$ as a result. Water vapor pressure decreases most rapidly at high temperatures. Air moisture content $\left(\mathrm{g} / \mathrm{m}^{3}\right)$ at $20{ }^{\circ} \mathrm{C}$ is about $1 / 30$ of that at the boiling point. This means that 29/30 of available water vapor have condensed over the distance from the location of boiling to the location of the $20^{\circ} \mathrm{C}$ contour. Hence, most of the porosity impacts are greatest in warm areas of the model domain. Drying of nodes near the heat source induces capillary wicking of brine to replace the water that has evaporated and drives a continuous cycle. Salt saturated water may also move into colder areas of the model where solubility is lower, causing precipitation of salt. This effect competes with condensation-induced dissolution of salt. The precise porosity change at any given node is therefore driven both by the change in total water volume and the temperature-dependent solubility of salt, both of which focus porosity increases in warm but sub-boiling areas of the model.

We compare results for porosity, saturation, volumetric water content as the product of porosity $\times$ saturation, and temperature (Fig. 5) through the horizontal centerline of the cylinder. Porosity results follow a similar pattern between the two retention functions, with a decrease near the heat source and an increase in the middle of the cylinder where abundant water condenses. However, results using the new retention function show a much more subdued increase in porosity, with a maximum value of about 0.47 compared to total dissolution when using the fixed, saturation-only retention function. Saturation results also contrast between the functions. With the fixed retention function, capillary pressure balance 


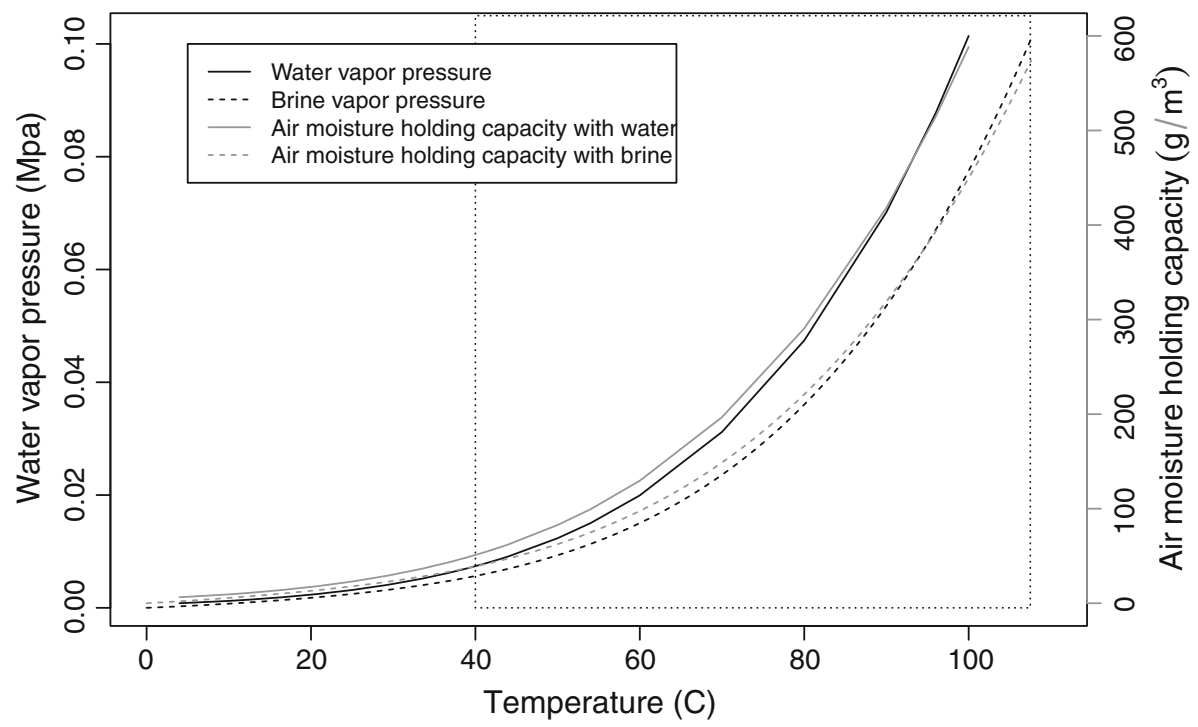

Fig. 4 Schematic of vapor pressure curve (black) and air moisture holding capacity (gray) for pure water (solid line) and brine (dashed line). Evaporation/condensation and precipitation/dissolution reactions are strongest in the steepest parts of the curve (rectangle)

is achieved by equilibrating saturation throughout the domain. When using the new retention function, where capillary pressure drops as porosity increases, pressure equilibrates at different saturations for different porosities. Specifically, high-porosity nodes must have lower saturation and low-porosity nodes must have higher saturation in order to balance capillary pressure.

As a consequence of the combination different porosity and different saturation, the two functions produce results that show highly contrasting volumetric water content. In both cases, the extremely low porosity at the heater causes low water content, but otherwise the two functions produce different outputs throughout the model domain. With equal saturation throughout, results using the saturation-only function show water content as following porosity, with most of the water concentrated in the highly porous nodes and reductions elsewhere in the domain. The porosity-dependent retention function, by contrast, causes nodes within the strong dissolution zone to be relatively dry, while water content is higher toward the cold end of the cylinder.

Temperature results for the two simulations show a marked difference in the distribution and gradients of temperatures. Total dissolution of a portion of the model domain in the simulation using the saturation-only retention function slows heat transfer through those nodes because the thermal conductivity function (Eq. 19) is truncated to a low value $(\sim 0.5 \mathrm{~W} / \mathrm{m} \mathrm{K})$. Heat transfer in this case is dominated by movement of air and vapor and by conduction through the brine in the pore space. As a result, heat transfer is very slow through the dissolution band and a steep thermal gradient is induced. By contrast, the simulation using the porosity-dependent retention function does not allow for total dissolution at any nodes. Thermal conductivity of the remaining solid fraction remains relatively high, so that the thermal gradient is shallower and temperatures at any given point in the domain are lower that at the same point in the fixed retention function simulation. 
(A)

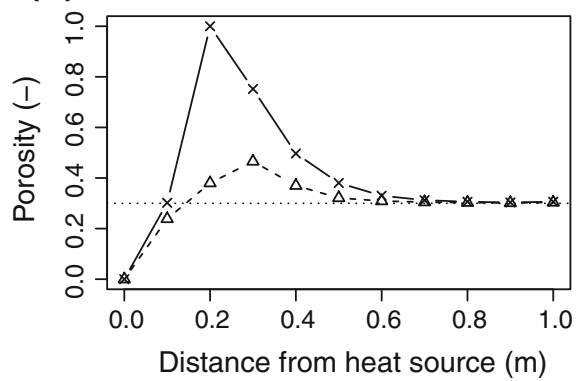

(C)

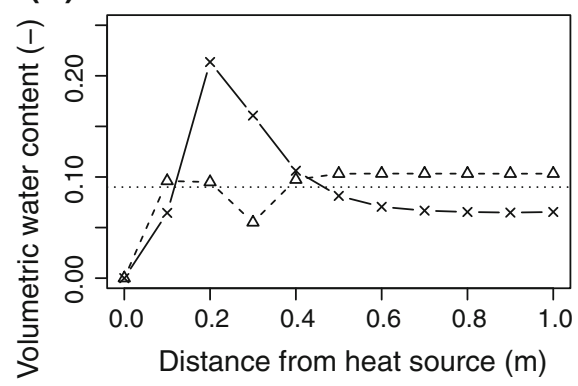

(B)

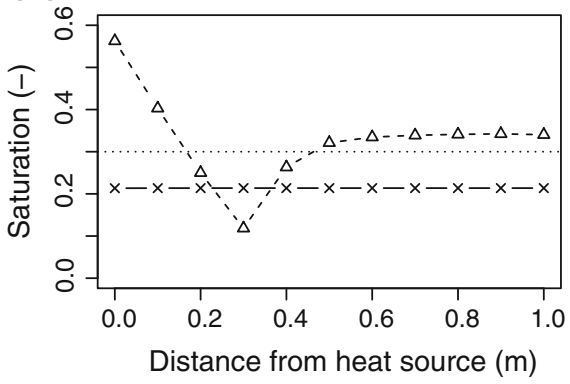

(D)

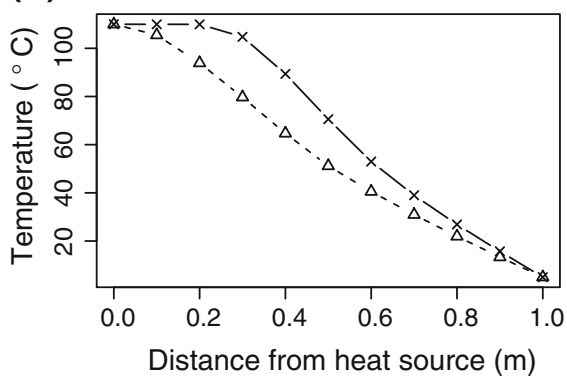

Initial state

$*$ Saturation-only model

- $\Delta$ - Porosity-dependent model

Fig. 5 365-day model results for porosity (a), saturation (b), water content (c), and Temperature (d). Initial condition is shown with dotted line. " $\mathrm{X}$ " symbols indicate results when a saturation-only retention function is used. Triangles indicate results with the new porosity-dependent retention function. Heat source is at $x=0$ and cold boundary is at $x=1$

\subsection{Square Box}

The second example scenario uses a $10 \mathrm{~m} \times 10 \mathrm{~m}, 2$-D square domain with $0.1 \mathrm{~m}$ spacing (Fig. 6). As previously described, we apply the salt function built within FEHM with porous media characteristics similar to the previous run. Boundary and initial conditions are designed to induce strong changes in porosity. The bottom $8 \mathrm{~m}$ is fully saturated at hydrostatic pressure, while the top $2 \mathrm{~m}$ is fully dry at time $t=0$. Initial temperature in the entire domain is $20^{\circ} \mathrm{C}$. The edges of the domain are no-flow boundaries with respect to mass. The top and right boundaries are held constant at $20^{\circ} \mathrm{C}$. A constant temperature of $120^{\circ} \mathrm{C}$ is input $3 \mathrm{~m}$ above the origin, and gravity is enabled in this numerical experiment. The geometry of the simulation causes convective circulation. Liquid recharge at the heat source is driven by capillary pull of water from as the water near the heat source boils and increases capillary pressure near the heater. Gravitational settling of water from higher in the domain also contributes to recharge near the heater. Strong porosity changes are induced by circulating flow of vapor and liquid. The setup of this simulation allows examination of the behavior of highly porous nodes above the saturated water surface. 400 days of model time are simulated for each scenario.

Results for porosity, saturation, and volumetric water content are shown in Fig. 7. For both retention functions, a low-porosity rind forms near the heat source. A dissolution front forms farther from the heat source (typically about $6 \mathrm{~m}$ ) where condensation and increased 


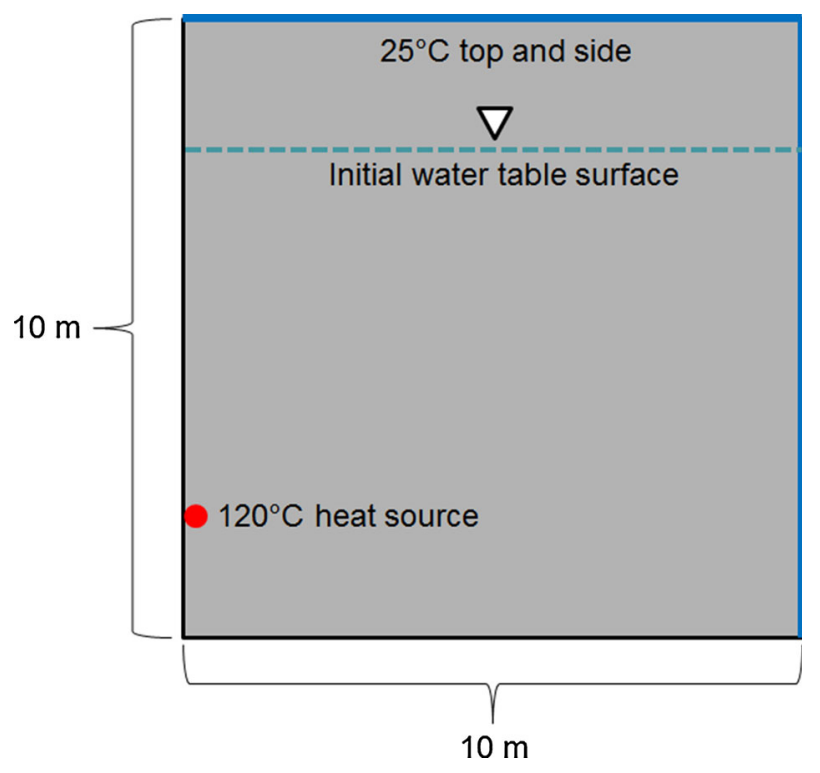

Fig. 6 Model domain for 2D setup. The box is closed with respect to mass flow. The top and right-hand boundaries are held constant at $25^{\circ} \mathrm{C}$. A constant $120^{\circ} \mathrm{C}$ temperature is input above the lower left corner, allowing for capillary draw of any water that accumulates below the heat source
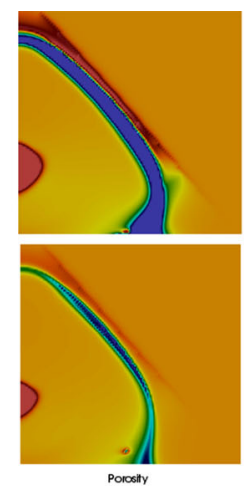
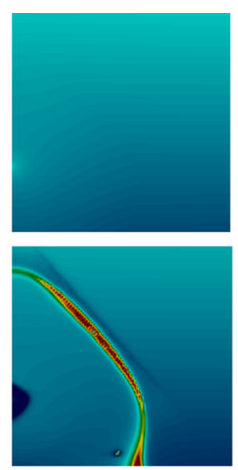

Saturation
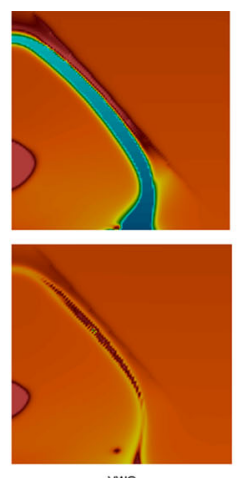

vwC

0.8
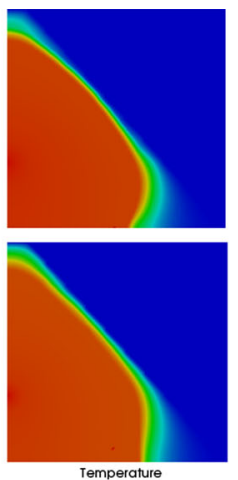

Temperature

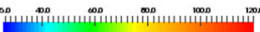

Fig. 7 (left to right) Porosity, saturation, volumetric water content (VWC), and Temperature $\left({ }^{\circ} \mathrm{C}\right)$ contours for the saturation-only retention function (top row) and new porosity-dependent retention function (bottom row). Porosity changes are restricted in the new function because capillary pull toward the heat source is prevented by the increasing porosity region which limits capillary pressure and residual saturation. Saturation is much more heterogeneous and water is drawn into lower-porosity regions surrounding the dissolution band. As a result, water content of the porous portions of the domain is greatly reduced in the new function, compared to very high water content (and retained water) in the wide dissolution band of the saturation-only retention function

solubility of salt combine for the greatest porosity change. However, using the new retention function, the dissolution front is slightly closer to the heat source and considerably narrower. Dissolution is weaker inside the dissolution band, with total dissolution only in a 1-2 node wide band that extends from about 3 to $8 \mathrm{~m}$ high in the box. 
Beyond the dissolution front is a secondary band of reduced porosity, which is wide and strongly reduced when using the saturation-only retention function. This same reduced porosity band is thin and of lower intensity when using the porosity-dependent retention function. The temperature gradient at this point is very sharp for both retention functions; consequently, nearly all of the water vapor condenses in the high-porosity region where water vapor pressure changes most rapidly. Little water vapor condenses beyond the dissolution front, so most of the water entering these nodes is drawn from the porous region in the form of liquid water that is already saturated with salt. Cooling causes the water to become oversaturated and salt to precipitate, closing pore space.

When the saturation-only retention function is applied, the dominance of capillary effects with the limited available volume of water causes saturation to be distributed nearly evenly through all nodes. A slight decrease is observed at the heat source due to repeated boiling of water, as well as a hydrostatic effect with more water at the bottom of the domain. Highporosity nodes in stacked vertical arrays remain partly saturated, highlighting the problem with water being retained in void space, the issue that originally motivated the new porositydependent retention function. By comparison, the new retention function has considerable heterogeneity in saturation. A band of near-zero saturation forms where high-porosity nodes drain into underlying nodes. Water is no longer retained in void space in these areas. Saturation is also low in the high-porosity nodes near the base of the model because of capillary drainage into the surrounding nodes. Meanwhile, low-porosity nodes in the cooling region and near the heat source have higher saturation than the corresponding parts of the saturation-only retention function simulation due to the much stronger capillary pressure in these nodes using the porosity-dependent form of the retention function.

As with the three-phase heat pipe numerical experiments described in Sect. 5.1, volumetric water content shows a nearly complete inversion in the high-porosity nodes when using the new retention function. The even distribution of saturation in the saturation-only retention function causes the highly porous areas to be water-rich, with up to $80 \%$ of the represented volume filled with water. Conversely, the new retention function preferentially drains highporosity nodes either into surrounding nodes through capillary flow or downward by gravity. Consequently, the porous nodes in the simulation using the porosity-dependent retention function have very low water contents while the high-porosity nodes in the simulation using the fix, saturation-only retention function have relatively high water content.

\section{Discussion}

The two thought experiments presented above demonstrate a fundamental change in the behavior of simulated three-phase heat pipes when a porosity-dependent retention function is included. When a single, saturation-only retention function is specified, pressure equilibrium is achieved by evenly distributing saturation throughout the domain; when capillary pressure is a major component of the pressure term in the system, the even distribution of saturation follows naturally from having a single retention function. As a result, water content scales with porosity, so that highly porous nodes contain large volumes of water. By contrast, the porosity-dependent retention function must vary saturation between nodes to equilibrate pressure; highly porous nodes must be dryer to have the same capillary pressure as lowporosity nodes. In fact, as porosity approaches one, capillary pressure goes to zero, resulting in no capillary pull of water toward open space. Based on our thought experiments, we plan to pursue real-world experiments to validate these concepts. 
The contrast in capillary behavior affects all types of results. Since the considered system is driven by reactions between the salt medium and water, variability in water content causes a feedback in the driving chemical reactions. In simulations using the new porosity-dependent retention function, this difference manifests as a limitation on the magnitude of porosity changes, where drying of nodes with increased porosity limits further dissolution, while the saturation-only retention function forces ever-stronger changes. Water content is inverted between simulations using the two retention functions. Differences in dissolution can force changes in heat transfer due to variation in thermal conductivity and convective moisture flow. The brine migration-driven aspects of the chemical reactions are also different between the two functions, because high-porosity regions limit capillarity-driven recharge of water to the heat source; especially in the salt cylinder model. This effect is important because of the lack of gravity flow to counter reduced capillary pressure.

The porosity-dependent retention function tends to induce an increase in the saturation of the lowest-porosity nodes when compared to the saturation-only retention function. In our salt cylinder thought experiment, the saturation of the lowest-porosity node nearly tripled when porosity effects on capillary pressure were taken into account. It may be further noted that presenting volumetric water content in the absence of saturation may lead to confusion as to the presence of water within low-porosity nodes because the porosity term dominates the saturation term, for example, a near-zero volumetric water content node can have high saturation. For applications in which the saturation in pore space matters, such as calculations of relative permeability, this may lead to erroneous interpretation of model results. Furthermore, direct examination of saturation results may aid in interpreting model behavior as compared to experimental results. In any case, for simulations of porous flow with changing porosity, presenting saturation data separately from volumetric water content and porosity may be useful for understanding the system behavior.

\section{Conclusion}

We developed a new retention function that dynamically alters the capillary pressure curve for porous media that experience porosity changes. A maximum pressure and residual saturation are calculated at each timestep based on the updated node porosity. Capillary pressure is then calculated as a function of saturation based on a new capillary pressure curve ranging between residual saturation and a maximum saturation, $0 \mathrm{MPa}$ pressure endpoint. The new function results in lower capillary pressures for high-porosity nodes at equal saturation as compared to low-porosity nodes, simulating stronger retention in tighter pore spaces and more complete drainage of open pores.

Two thought experiments presented in this paper illustrate how accounting for porositydependent retention alters simulation results and the fundamental behavior of the system. Fixed, saturation-only retention functions tend to equilibrate saturation evenly throughout the domain, resulting in unphysical liquid water retained in void space. Water therefore tends to accumulate in high-porosity regions of the model domain. The presence of abundant water in highly porous nodes can further strengthen dissolution/precipitation reactions in heat pipe settings due to heating and continued flow of brine toward the heat source. The porosity-dependent retention function tends to instead draw water out of higher porosity regions into adjacent low-porosity regions where capillary pressures are stronger for equal saturation. As local porosity increases, water will drain from these nodes. If sufficient porosity is attained, further flow is prevented and the heat pipe slows or stops. These two modes cause 
fundamental differences in simulation outputs, especially in a system such as the numerical thought experiments in salt where chemical reactions driven in part by moisture content lead to strong three-phase heat pipes.

Based on our numerical thought experiments, large model errors may be produced if fixed, saturation-only retention functions are applied to simulations of porous media with changing porosity. We observed large differences in results for porosity, saturation, volumetric water content, temperature, and even the location at which porosity changes occur when we compared simulations using the fixed, saturation-only retention function to those using the porosity-dependent retention function. These errors may be particularly problematic in scenarios where capillary effects are an important driver of the porosity changes. A porositydependent retention function, such as the one developed here, can improve simulation results in such cases.

Acknowledgements This work was undertaken in support of research funded by the DOE Office of Nuclear Energy, on grant DMS SFWD-SFWST-2017-000102.

Open Access This article is distributed under the terms of the Creative Commons Attribution 4.0 International License (http://creativecommons.org/licenses/by/4.0/), which permits unrestricted use, distribution, and reproduction in any medium, provided you give appropriate credit to the original author(s) and the source, provide a link to the Creative Commons license, and indicate if changes were made.

\section{References}

Arnold, B.A., Kuzio, S.P., Robinson, B.A.: Radionuclide transport simulation and uncertainty analyses with the saturated-zone site-scale model at Yucca Mountain, Nevada. J. Contam. Hydrol. 62-63, 401-419 (2003)

Ball, J.L., Stauffer, P.H., Calder, E.S., Valentine, G.A.: The hydrothermal alteration of cooling lava domes. Bull. Volcanol. 77, 102 (2015). https://doi.org/10.1007/s00445-015-0986-Z

Bechtold, W., Smallos, E., Heusermann, S., Bollingerfehr, W., Bazargan-Sabet, B., Rothfuchs, T., Kamlot, P., Gnupa, J., Olivella, S., Hansen, F.D.: Backfilling and sealing of underground repositories for radioactive waste in salt (BAMBUS II Project). EUR 20621 EN, European Commission (2004)

Birdsell, K.H., Wolfsberg, A.V., Hollis, D., Cherry, T.A., Bower, K.M.: Groundwater flow and radionuclide transport calculations for a performance assessment of a low-level waste site. J. Contam. Hydrol. 46, 99-129 (2000)

Birkholzer, J.T.: Estimating liquid fluxes in thermally perturbed fractured rock using measured temperature profiles. J. Hydrol. 327(3-4), 496-515 (2004). https://doi.org/10.1016/j.jhydrol.2005.11.049

Boudreau, B.P., Bennett, R.H.: New rheological and porosity equations for steady-state compaction. Am. J. Sci. 299, 517-528 (1999)

Bourret, S.M., Johnson, P.J., Zyvoloski, G.A., Chu, S.P., Weaver, D.J., Otto, S., Boukhalfa, H., Caporuscio, F.A., Jordan, A.B., Stauffer, P.H.: Experiments and modeling in support of generic salt repository science. Los Alamos National Laboratory, USDOE Used Fuel Disposition Campaign Final Report, LA-UR-1627329 (2016)

Bourret, S.M., Otto, S., Johnson, P.J., Weaver, D.J., Boukhalfa, H., Stauffer, P.H.: High level waste in salt repositories: experiments and simulations of evaporation in the underground. In: Proceedings of 2017 Waste Management (2017)

Brandt, K.A., Goldman, S.A., Inglin, T.A.: Hydrogel-forming polymer compositions for use in absorbent structures. Patent, US RE362649 E (1987)

Brooks, R.H., Corey, A.T.: Hydraulic properties of porous media. Hydrology Papers, Colorado State University, Fort Collins, Colorado (1964)

Buckles, R.S.: Correlating and averaging connate water saturation data. J. Can. Pet. Technol. 9(1), 42-52 (1965)

Calore, C., Battistelli, A.: Application of TOUGH2/EWASG to the modelling of salt water injection into a depleted geothermal reservoir: preliminary results. In: Conference Paper, TOUGH Symposium 2003, Berkeley, CA, USA Proceedings (2003) 
Caporuscio, F.A., Boukhalfa, H., Cheshire, M.C., Jordan, A.B., Ding, M.: Brine migration experimental studies for salt repositories. FCRD Used Fuel Disposition Campaign Milestone FCRD-UFD-2013-000204 (2013)

Cinar, Y., Pusch, G., Reitenbach, V.: Petrophysical and capillary properties of compacted salt. Transp. Porous Media 65(2), 199-228 (2006)

Doughty, C., Pruess, K.: A similarity solution for two-phase fluid and heat flow near high-level nuclear waste packages emplaced in porous media. Int. J. Heat Mass Transf. 33(6), 1205-1222 (1990)

Doughty, C.: Users guide for hysteretic capillary pressure and relative permeability functions in TOUGH2. Report, Lawrence Berkeley National Laboratory, Berkeley, CA, LBNL-6533E (2013)

Evans, R.L., Lizarralde, D.: Geophysical evidence for karst formation associated with offshore groundwater transport: an example from North Carolina. Geochem. Geophys. Geosyst. 4(8), 1069-1078 (2003)

Fakcharoenphol, P., Xiong, Y., Hu, L., Winterfield, P.H., Xu, T., Wu, Y.: User's guide of TOUGH2-EGS: a coupled geomechanical and reactive geochemical simulator for fluid and heat flow in enhanced geothermal systems, version 1.0. Petroleum Engineering Department, Colorado School of Mines (2013)

FEHM: FEHM Website. https://fehm.lanl.gov/. Accessed 11 Jan 2017

Finn, R.: Capillary surface interfaces. Not. AMS 46(7), 770-781 (1999)

Fisher, A.T., Von Herzen, R.: Models of hydrothermal circulation within 106 Ma seafloor: constraints on the vigor of fluid circulation and crustal properties below the Madeira Abyssal Plain. Geochem. Geophys. Geosyst. (2005). https://doi.org/10.1029/2005gc001013

Gable, C.W., Clayton, D.J., Lu, Z.: Inverse modeling to determine thermal properties of salt due to heating from high level waste emplaced in a generic salt repository. US DOE Office of Nuclear Fuel Recycling Report AFCI-WAST-PMO-DV-2009-000001 (2009)

Gallipoli, D., Wheeler, S., Karstunen, M.: Modelling of the variation of degree of saturation in a deformable unsaturated soil. Géotechnique 53(1), 105-112 (2003)

Gingras, M.K., Baniak, G., Gordon, J., Hovikoski, J., Konhauser, K.O., La Croix, A., Lemiski, R., Mendoza, C., Pemberton, S.G., Polo, C., Zonneveld, J.: Porosity and permeability in bioturbated sediments. Dev. Sedimentol. 64, 837-867 (2012). https://doi.org/10.1016/B978-0-444-53813-0.00027-7

Gluyas, J., Coleman, J.: Material flux and porosity changes during sediment diagenesis. Nature 356(6364), 52-54 (1992)

Haar, L., Gallagher, J.S., Kell, G.S.: NBS/NRC Steam, Tables. Hemisphere, New York (1984)

Harp, D.R., Stauffer, P.H., Mishra, P.K., Levitt, D.G., Robinson, B.A.: Modeling of high-level nuclear waste disposal in a salt repository. Nucl. Technol. 187, 294-307 (2014). https://doi.org/10.13182/NT13-110

Ho, C., Webb, S.: Review of porous media enhanced vapor-phase diffusion mechanisms, models, and data: Does enhanced vapor-phase diffusion exist? J. Porous Media 1, 71-92 (1998)

Holmes, M., Holmes, A., Holmes, D.: Relationship Between Porosity and Water Saturation: Methodology to Distinguish Mobile from Capillary Bound Water. AAPG Annual Convention and Exhibition, Denver, CO (2009)

Jo, H.S., An, S., Nguyen, X.H., Kim, Y.I., Bang, B., James, S.C., Choi, J., Yoon, S.S.: Modifying capillary pressure and boiling regime of micro-porous wicks textured with graphene oxide. Appl. Therm. Eng. 128(5), 1605-1610 (2018). https://doi.org/10.1016/j.applthermaleng.2017.09.103

Johnson, P.J., Bourret, S.M., Boukhalfa, H., Caporuscio, F.A., Zyvoloski, G.A., Weaver, D.J., Otto, S., Stauffer, P.H.: Experiments and modeling to support field test design. Los Alamos National Laboratory, USDOE Fuel Cycle Research \& Development Final Report, LA-UR-17-27759 (2017)

Jordan, A.B., Boukhalfa, H., Caporuscio, F.A., Stauffer, P.H.: Brine Transport Experiments in Run-of-Mine Salt. Los Alamos National Laboratory Report, LA-UR-15-26804 (2015a)

Jordan, A.B., Zyvoloski, G.A., Weaver, D.J., Otto, S., Stauffer, P.H.: Coupled thermal-hydrologic-chemical model for in-drift disposal test. Los Alamos National Laboratory Report, LA-UR-15-27442 (2015b)

Jordan, A.B., Boukhalfa, H., Caporuscio, F.A., Robinson, B.A., Stauffer, P.H.: Hydrous mineral dehydration around heat-generating nuclear waste in bedded salt formations. Environ. Sci. Technol. 5, 1-13 (2015). https://doi.org/10.1021/acs.est.5b01002

Jury, W.A., Gardner, W.R.: Soil Physics, 5th edn. Wiley, New York (1991)

Kelkar, S., Tucci, P., Srinivasan, G., Roback, R., Robinson, B., Duke, C., Rehfeldt, K.: Breakthrough of radioactive plumes in saturated volcanic rock: implications from the Yucca Mountain Site. Geofluids 13(3), 273-282 (2013). https://doi.org/10.1111/gfl/12035

Kolditz, O., Bauer, S., Bilke, L., Böttcher, N., Delfs, J.O., Fischer, T., Görke, U.J., Kalbacher, T., Kosakowwski, G., McDermott, C.I., Park, C.H., Radu, F., Rink, K., Shao, H., Shao, H.B., Sun, F., Sun, Y.Y., Singh, A.K., Taron, J., Walther, M., Wang, W., Watanabe, N., Wu, Y., Xie, M., Xu, W., Zehner, B.: OpenGeoSys: an open-source initiative for numerical simulation of thermos-hydro-mechanical/chemical (THM/C) processes in porous media. Environ. Earth Sci. (2012). https://doi.org/10.1007/s12665-012-1546-X 
Kuhlman, K.L., Malama, B.: Brine flow in heated geologic salt. Sandia National Laboratories Report SAND2013-1944 (2013)

Leverett, M.C.: Capillary behavior in porous solids. Soc. Pet. Eng. 142(1), 152-169 (1941). https://doi.org/1 $0.2118 / 941152-\mathrm{g}$

Lichtner, P.C., Hammond, G.E., Lu, C., Karra, S., Bisht, G., Andre, B., Mills, R., Kumar, J.: A massively parallel reactive flow and transport model for describing surface and subsurface processes. PFLOTRAN user manual (2015). http://www.pflotran.org/docs/user_manual.pdf

Lucas, R.: Rate of capillary ascension of liquids. Kollid Z. 23, 15-22 (1918)

Masoodi, R., Pillai, K.M.: A general formula for capillary suction-pressure in porous media. J. Porous Media 15(8), 775-783 (2012). https://doi.org/10.1615/JPorMedia.v15.i8.60

Millington, R.J., Quirk, J.P.: Permeability of porous solids. Trans. Faraday Soc. 57, 1200-1207 (1961)

Munson, D.E., Jones, R.L., Ball, J.R., Clancy, R.M., Hoag, D.L., Petney, S.V.: Overtest for simulated defense high-level waste (Room B): In situ data report (May 1984-February 1988): Waste Isolation Pilot Plant (WIPP) Thermal/Structural Interactions Program. Sandia National Labs Technical Report SAND-892671 (1990)

Nuth, M., Laloui, L.: Advances in modelling hysteretic water retention function in deformable soils. Comput. Geotech. 35(6), 835-844 (2008). https://doi.org/10.1016/j.compgeo.2008.08.001

Oh, S., Lu, N.: Uniqueness of the suction stress characteristic curve under different confining stress conditions. Vadose Zone J. (2014). https://doi.org/10.2136/vzj2013.04.0077

Olivella, S., Castagna, S., Alonso, E.E., Lloret, A.: Porosity variations in saline media induced by temperature gradients: experimental evidences and modeling. Transp. Porous Media 90(3), 763-777 (2011)

Pérès, G., Cluzeau, D., Curmi, P., Hallaire, V.: Earthworm activity and soil structure changes due to organic enrichments in vineyard systems. Biol. Fertil. Soils 27(4), 417-424 (1998)

Pruess, K.: TOUGH2 - a general-purpose numerical simulator for multiphase fluid and heat flow. Lawrence Berkeley Laboratory LBL-29400 (1991)

Rutqvist, J.L., Martin, B., Molins, S., Trebotich, D., Birkholzer, J.: Modeling coupled THM processes and brine migration in salt at high temperatures. UFD Document FCRD-UFD-2015-000366, LBNL-191216 (2016)

Salager, S., El Youssoufi, M.S., Saix, C.: Definition and experimental determination of a soil-water retention surface. Can. Geotech. J. 47(6), 609-622 (2010). https://doi.org/10.1139/T09-123

Šimůnek, J., van Genuchten, M.T., Šejna, M.: HYDRUS: model use, calibration, and validation. Trans. Am. Soc. Agric. Eng. 55(4), 1261-1274 (2012). https://doi.org/10.13031/2013.42239

Sparrow, B.S.: Empirical equations for the thermodynamic properties of aqueous sodium chloride. Desalination 159(2), 161-170 (2003). https://doi.org/10.1016/S0011-9164(03)90068-3

Spinelli, G.A., Fisher, A.T.: Hydrothermal circulation within rough basement on the Juan de Fuca Ridge flank. Geochem. Gephys. Geosyst. 5(2), Q02001 (2004). https://doi.org/10.1029/2003GC000616

Stauffer, P.H., Auer, L.H., Rosenberg, N.D.: Compressible gas in porous media: a finite amplitude analysis of natural convection. Int. J. Heat Mass Transf. 40(7), 1585-1589 (1997)

Stauffer, P.H., Birdsell, K.H., Witkowski, M.S., Hopkins, J.K.: Vadose zone transport of 1,1,1-trichloroethane. Vadoze Zone J. 4(3), 760-773 (2005)

Stauffer, P.H.: Flux flummoxed: a proposal for consistent usage. Ground Water 44(2), 125-128 (2006)

Stauffer, P.H., Vrugh, J.A., Turin, H.J., Gable, C.W., Soll, W.E.: Untangling diffusion from advection in unsaturated porous media: experimental data, modeling, and parameter uncertainty. Vadose Zone J. 8(2), 510-522 (2009). https://doi.org/10.2136/vzj2008.0055

Stauffer, P.H., Harp, D.R., Jordan, A.B., Lu, Z., Kelkar, S., Kang, Q., Ten Cate, J., Boukhalfa, H., Labyed, Y., Reimus, P.W., Caporuscio, F.A., Miller, T.A., Robinson, B.A.: Coupled model for heat and water transport in a high level waste repository in salt. Los Alamos National Laboratory, DOE Level 2 Milestone FCRDUFD-2013-000206, LA-UR-13-27584 (2013)

Stauffer, P.H., Jordan, A.B., Lu, Z., Zyvoloski, G.A., Boukhalfa, H., Caporuscio, F.A., Miller, T.A., Robinson, B.A.: Thermo-hydrological and chemical (THC) modeling to support field test design. Los Alamos National Laboratory Document, Milestone M4FT-14LA0818064, LA-UR 14-27548 (2014a)

Stauffer, P.H., Lewis, K.C., Stein, J.S., Travis, B.J., Lichtner, P., Zyvoloski, G.A.: Joule-Thomson effects on the flow of liquid water. Transp. Porous Media (2014). https://doi.org/10.1007/s11242-014-0379-3

Sun, Y., Kharaghani, A., Metzger, T., Müller, J., Tsotsas, E.: Lotion distribution in wet wipes investigated by pore network simulation and X-ray micro tomography. Transp. Porous Media 107(2), 449-468 (2015)

Sweijen, T., Nikooee, E., Hassanizadeh, S.M., Chareyre, B.: The effects of swelling and porosity change on capillarity: DEM coupled with a pore-unit assembly method. Transp. Porous Media 115, 207-226 (2016). https://doi.org/10.1007/s11242-016-0689-8 
Tenma, N., Yamaguchi, T., Zyvoloski, G.: The Hijiori Hot Dry Rock test site, Japan: evaluation and optimization of heat extraction from a two-layered reservoir. Geothermics 37, 19-52 (2008). https://doi.org/10.1016/ j.geothermics.2007.11.002

Tsang, C.: Linking thermal, hydrological, and mechanical processes in fractured rocks. Annu. Rev. Earth Planet. Sci. 27, 359-384 (1999). https://doi.org/10.1146/annurev.earth.27.1.359

Van Genuchten, M.T.: A closed-form equation for predicting the hydraulic conductivity of unsaturated soils. Soil Sci. Soc. Am. J. 44(5), 892-898 (1980)

Waltham, T., Bell, F.G., Culshaw, M.G.: Rocks, Dissolution, and Karst, in Sinkholes and Subsidence. Karst and Cavernous Rocks in Engineering and Construction. Springer, Berlin (2005). https://doi.org/10.100 7/3-540-26953-3_1

Wang, W., Fischer, T., Zehner, B., Böttcher, N., Görke, U.J., Kolditz, O.: A parallel finite element method for two-phase flow processes in porous media: OpenGeoSys with PETSc. Environ. Earth Sci. 73(5), 2269-2285 (2015). https://doi.org/10.1007/s12665-014-3576-z

Washburn, E.V.: The dynamics of capillary flow. Phys. Rev. 17, 273-283 (1921)

Winslow, D.M., Fisher, A.T., Stauffer, P.H., Gable, C.W., Zyvoloski, G.A.: Three-dimensional modeling of outcrop-to-outcrop hydrothermal circulation on the eastern flank of the Juan de Fuca Ridge. J. Geophys. Res. Solid Earth 121(3), 1365-1382 (2016). https://doi.org/10.1002/2015jb012606

Yamaguchi, T., Kunyagawa, M., Sato, Y., Oikawa, Y., Kobayashi, H., Matsunaga, I., Zyvoloski, G.: Heat extraction test from hot dry rock and reservoir modeling. J. Geotherm. Res. Soc. Jpn. 13, 73-93 (1991)

Zyvoloski, G.A., Robinson, B.A., Dash, Z.V., Kelkar, S., Viswanathan, H.S., Pawar, R.J., Stauffer, P.H., Miller, T.A., Chu, S.P.: Software users manual (UM) for the FEHM Application Version 3.1-3.X, LANL Report, LA-UR-12-24493 (2012) 